\title{
DISCHARGE MODULATION NOISE IN He-Ne LASER RADIATION
}

\author{
P. T. BOLWIJN \\ Physics Laboratory, State University Utrecht, The Netherlands
}

Received 19 January 1967

\begin{abstract}
Discharge modulation noise in He-Ne laser radiation is considered theoretically, including explicitly the laser oscillator properties. Experiments reported previously by us and other authors are in agreement with our analysis.
\end{abstract}

The radiation of $d . c$. excited He-Ne lasers may show a large amount of low-frequency noise [1] which is due to modulation of the internal laser field by discharge current fluctuations [2-7]. Here we give an analysis of this modulation noise which includes explicitly the laser oscillator properties as derived by Lamb [8], in contrast to the analysis given by other authors $[5,7]$.

As a result of discharge current fluctuations the number of excited metastable He atoms and $\mathrm{Ne}$ atoms in the upper laser level fluctuate. This fluctuation in the driving force of the laser oscillation may be described by including a stochastic, slowly varying term $\Delta N(t)$ in $N$, which is defined by Lamb [8] as the excitation density in absence of optical oscillations. Thus we write $N=\bar{N}+$ $+\Delta N(t)$, with $\Delta N(t) \ll \bar{N}$. Using Lamb's differential equation for the amplitude $E(t)$ of the electric field in the laser interferometer $\mathrm{d} E / \mathrm{d} t=\alpha E-\beta E^{3}$, and its steady-state solution, $E_{\mathrm{o}}{ }^{2}=\alpha / \beta$, we find for the fluctuating part $\Delta E(t)$ in $E$ [cf. 9,10]

$$
\mathrm{d} \Delta E / \mathrm{d} t+2 \alpha \Delta E=\left(\frac{1}{2} \nu / Q\right)\left(E_{\mathrm{O}} / \bar{N}\right) \Delta N(t),
$$

where we assumed $\Delta E(t) \ll E_{0}$. The quantities and $\beta$ respresent the unsaturated net gain and the saturation, respectively, as defined by Lamb [8].

Eq. (1) is a typical Langevin equation wellknown in noise analysis. The relation between the spectral noise intensities $S_{\Delta N}(f)$ and $S_{\Delta E}(f)$ can easily be found by making a Fourier analysis of eq. (1). The spectral noise intensity of the laser power output $\propto E^{2}$ follows from $S_{\Delta\left(E^{2}\right)}(f)=$ $=4 E_{0}{ }^{2} S_{\Delta E}(f)$. We have

$s_{\Delta\left(E^{2}\right)}(f)=\left(\frac{1}{2} \nu / Q\right)^{2} S_{\Delta N}(f) / \bar{N}^{2}{ }_{\beta}^{2}\left[1+(\omega / 2 \alpha)^{2}\right]$,

where $\omega=2 \pi f$. We assumed implicitly that $f$ is much smaller than the decay rates of the laser levels ( $f \ll 10 \mathrm{Mc} / \mathrm{s}$ in case of He-Ne lasers).
It is seen from eq. (2) that for a given $S_{\Delta N}(f)$ the intensity fluctuations decrease with increasing average excitation density $\bar{N}$ and saturation $\beta$, as may be expected. The saturation of a gas laser transition is known to become stronger near atomic line center [8]. It follows then from eq. (2) that for $\omega \ll 2 \alpha$ the noise intensity of a singlemode gas laser versus interferometer detuning should show a dip near line center, which we observed indeed [4]*. In ref. 4 also reported the noise intensity near threshold detuning varied proportionally to the square of the d.c. laser output with varying interferometer detuning. Close to threshold we have $\omega \gg 2 \alpha$ [cf. 10], so that according to eq. (2) $S_{\Delta}\left(E^{2}\right)(f) \propto(\alpha / \beta)^{2}=E_{\mathrm{O}} 4$, indeed.

In discussing the noise spectrum of the laser radiation we have also to consider the noise spectrum $S_{\Delta N}(f)$, being determined by relaxation effects in the discharge. Let us discuss briefly some factors which affect the noise spectrum. In case of $\mathrm{He}-\mathrm{Ne}$ lasers the slowest decay rate in the (de-)excitation processes involved is the inverse lifetime $\tau$ of the metastable He atoms $\left(2^{3} \mathrm{~S}\right.$ or $\left.2^{1} \mathrm{~S}\right)$ which at low electron densities describes the rate of energy transfer between He and the upper laser level [cf. 7]. The population of metastable $\mathrm{He}$ atoms may fluctuate because of fluctuations in the density $n_{\mathrm{e}}$ of electrons that have sufficient energy to excite the metastable $\mathrm{He}$ atoms. We may expect

$$
s_{\Delta N}(f) \propto s_{\Delta n_{e}}(f) /\left[1+(\omega \tau)^{2}\right],
$$

\footnotetext{
* Fluctuations in the laser radiation due to fluctuations in the optical quality of the interferometer would also show a similar central tuning dip $[10,11]$. This noise source, however, was certainly not predominant in our case, since it cannot explain the observed crosscorrelation between laser noise and discharge-current noise $[3-5]$.
} 
where $S_{\Delta n_{e}}(f)$ is the spectral noise intensity of $n_{\mathrm{e}}$ in the active region, which may be a rather complicated function due to the possible occurrence of many types of discharge instabilities [12] including the effect of cathode- and anode-oscillations and striations. The dependence of the fluctuations in the discharge current $i_{\mathrm{d}}$ on the fluctuations in $n_{\mathrm{e}}$ within and outside the active region may be described by the equivalent circuit of the gas discharge tube introduced in [5]. Direct information on $S_{\Delta N}(f)$ may be obtained from a noise analysis of the filtered side light [cf. 4].

Whether the cutoff-frequency $2 \alpha$ (i.e. the active interferometer width $[10,13])$ or $\tau^{-1}$ will appear in the laser noise spectrum measured, depends on the pasfive interferometer width, on the excitation level and, especially so in a single-mode laser, on the interferometer detuning. The values of $\tau$ are in typical'gas laser mixtures $10-50 \mu \mathrm{sec}$ (for $\mathrm{He} 2^{3} \mathrm{~S}$ ) and 1-5 $\mu \mathrm{sec}$ (for He $2^{1} \mathrm{~S}$ [14]. It can be shown that in most cases $\tau-1<2 \alpha$, except near threshold, and the dominant cutoff-frequency will then be $\tau^{-1}$, being largely independent of the laser operating conditions. Cutoff-frequencies in modulation noise spectra observed by us [4], see also [10] and others [3,5-7] are in general agree with these orders of magnitude. This was concluded independently also by Kubo et al. [6,7]. By Prescott et al. [5] the laser noise spectrum was observed to decay stronger at low excitation levels than at high excitation levels, which can be shown to be highly probably due to the effect of $2 \alpha$.

An important role in the determination of the intensity of the laser noise is played by the proportionally factor in eq. (3), which relates the fluctuations in $n_{\mathrm{e}}$ to those in $N$ at $f \approx 0$. This factor is connected to the slope of the function $N\left(n_{\mathrm{e}}\right)$. At high electron densities, for instance, this function may flatten out due to destructive collisions of metastable $\mathrm{He}$ atoms with electrons [15] or with other excited atoms [16]. Leaving other factors fixed and disregarding other possible fluctuations sources, such as the energy of the electrons, the laser noise intensity is expected to reduce due to this plateau.
We conclude that for a complete description of the noise in laser radiation due to gas discharge fluctuations, we have to consider the rate equations for the (de-)excitation processes involved [7], the equivalent circuit for the discharge im pedances [5] as well as the laser properties. It should be noted that at high excitation levels and at high gas pressures the Lamb equations used here are no longer valid, as shown by our laser modulation experiments [10]. A more detailed account will appear elsewhere [17].

The author is highly indebted to Prof. Dr.C. Th.J. Alkemade for numerous helpful discussions. He wishes to express his appreciation to the N.V. Philips' Gloeilampenfabrieken for supplying the laser.

\section{References}

1. P.T.Bolwijn, C.Th.J.Alkemade and G.A. Boschloo, Phys. Letters 4 (1963) 59.

2. J.A.Bellisio, C.Freed and H.A.Haus, Appl. Phys. Letters 4 (1964) 5.

3. L.J. Prescott and A. Van der Ziel, Appl. Phys. Letters 5 (1964) 48.

4. P.T.Bolwijn, Phys. Letters 13 (1964) 311.

5. L. J. Prescott and A. Van der Ziel, IEEE J. Quant. Elec. QE2 (1966) 173.

6. U.Kubo, K. Kawabe and Y.Inuishi, Japan J. Appl. Phys. 5 (1966) 731.

7. U.Kubo, K. Kawabe and Y.Inuishi, Techn. Rep. Osaka Univ. 16 (1966).

8. W.E.Lamb Jr., Phys. Rev. 134 (1964) A 1429.

9. P.T.Bolwijn, Phys. Letters 19 (1965) 384.

10. P.T.Bolwijn, IEEE J. Quant. Elec. QE2, (1966) 670; J. Appl. Phys. 37 (1966) 4487.

11. Th. H. Peek, P.T.Bolwijn and C.Th.J.Alkemade, Phys. Letters 24A (1967) 128.

12. F.W. Crawford and G.S.Kino, Proc. IEEE 49 (1961) 1767.

13. T. Uchida, IEEE J. Quant. Elec. QE1 (1965) 336.

14. N.Suzuki, Japan J. Appl. Phys. 3 (1964) 705.

15. A.D.White and E.I.Gordon, Appl. Phys. Letters 3 (1963) 197.

16. J.Y.Wada and H.Heil, Res. Rep. no. 337, Hughes Res. Labs. (June 1965).

17. P.T.Bolwijn, thesis, Utrecht, to be published. 